\title{
Comparison of Computed Tomography Dose Reporting Software
}

\section{A Abdullah ${ }^{1}, \mathrm{Z} \mathrm{Sun}^{2}, \mathrm{~N}$ Pongnapang ${ }^{3}, \mathrm{~K}-\mathrm{H} \mathrm{Ng}{ }^{4,5}$}

${ }^{1}$ Medical Physics, Comprehensive Cancer Center, King Fahad Medical City, PO Box 59046, 11525 Riyadh, Saudi Arabia

${ }^{2}$ Discipline of Medical Imaging, Department of Imaging and Applied Physics, Curtin University, Perth, Western Australia 6845, Australia

${ }^{3}$ Faculty of Medical Technology, Mahidol University, Bangkok 10700, Thailand

${ }^{4}$ Department of Biomedical Imaging, University of Malaya, 50603 Kuala Lumpur, Malaysia 5University of Malaya Research Imaging Centre, University of Malaya, 50603 Kuala Lumpur, Malaysia

\section{Corresponding author:}

Professor Kwan-Hoong Ng, Department of Biomedical Imaging, University of Malaya, 50603 Kuala Lumpur, Malaysia

Tel: 60379493930

Fax: 60379494603

Email: ngkh@ummc.edu.my 


\section{Abstract}

Computed tomography (CT) dose reporting software facilitates the estimation of doses to patients undergoing CT examinations. In this study, comparison of three software packages i.e. CT-Expo (version 1.5, Medizinische Hochschule, Hannover, Germany), ImPACT CT Patients Dosimetry Calculator (version 0.99x, Imaging Performance Assessment on Computed Tomography, www.impactscan.org) and WinDose (version 2.1a, Wellhofer Dosimetry, Schwarzenbruck, Germany) has been made in terms of their calculation algorithm and results of calculated doses. Estimations were performed for head, chest, abdominal and pelvic examinations based on the protocols recommended by the European guidelines using single-slice CT (SSCT) (Siemens Somatom Plus 4, Erlangen, Germany) and multi-slice CT (MSCT) (Siemens Sensation 16, Erlangen, Germany) for software-based female and male phantoms. The results showed that there are some differences in final dose reporting provided by these software packages. There are deviations of effective doses produced by these software packages. Percentages of coefficient of variance range from $3.3 \%$ to $23.4 \%$ in SSCT and $10.6 \%$ to $43.8 \%$ in MSCT. It is important that researchers state the name of the software that is used to estimate the various CT dose quantities. Users must also understand the equivalent terminologies between the information obtained from the CT console and the software packages in order to use the software correctly.

Keywords: computed tomography, dose reporting software, effective dose, organ dose 


\section{Introduction}

While the benefits of CT in medicine are well known, increasing concerns about the radiation dose associated with CT have captured the attention of imaging professionals, referring practitioners, general public and the news media. ${ }^{(1-3)}$ In June 2009, The American College of Radiology (ACR) and the Radiological Society of North America (RSNA) established the Joint Task Force on Adult Radiation Protection to address how dose optimisation could be incorporated into all imaging practices and the concept of radiation dose disseminated to the healthcare professionals. Efforts have taken place in recent years to increase awareness about adult and paediatric radiation protection. ${ }^{(4-6)}$ The medical imaging community must ensure that the benefits of a CT examination in any given patient outweighs the corresponding risks. (7)

Currently, it is a requirement that CT scanners display protocol information and selected dosimetry metrics such as volume CT dose index $\left(\mathrm{CTDI}_{\mathrm{vol}}\right)$ and dose-length product (DLP). ${ }^{(8,}$

9) This allows radiologists and medical imaging technologists to access valuable information about scanner performance and patient dose. Dose distribution in CT is different compared to projection radiography. Therefore, estimation of organ doses and effective dose in CT is more complicated than with projection radiography. A few studies have been done to simplify the estimation of effective dose from DLP. ${ }^{(10,11)}$ On the other hand, dose reporting software packages have been developed to facilitate the estimation of effective doses. ${ }^{(12-14)}$ These software packages have become very popular in recent times, because they are easy to use and give quick results. Users are required to select or insert appropriate CT parameters into the software in order to obtain comprehensive values of CT dose descriptors i.e. organ doses and effective dose for patients undergoing CT examinations. This is useful for optimisation of techniques. 
However, different institutions or clinical centres may use different software packages. There are differences among the software packages due to different methods and algorithms adopted by these software developers. As a result, dose values reported may differ depending on the software used. The purpose of this study is to compare three commonly used software packages. The comparison includes the software calculation methods and algorithms, and the values of estimated effective doses for some common CT examinations.

\section{Materials and Methods}

This study compares three common software packages i.e. CT-Expo (version 1.5, Medizinische Hochschule, Hannover, Germany), ImPACT CT Patients Dosimetry Calculator (version 0.99x, Imaging Performance Assessment on Computed Tomography, www.impactscan.org) and WinDose (version 2.1a, Wellhofer Dosimetry, Schwarzenbruck, Germany). Estimation of dose descriptors on the software packages i.e. weighted CTDI $\left(\mathrm{CTDI}_{\mathrm{w}}\right)$, volume CTDI (CTDI $\left.{ }_{\mathrm{vol}}\right)$, organ doses and effective dose, were made for single-slice CT (SSCT) (Siemens Somatom Plus 4, Erlangen, Germany) and multi-slice CT (MSCT) (Siemens Sensation 16, Erlangen, Germany).

CT-Expo and ImPACT Calculator cover most models of CT scanners available today but the WinDose provides a few selected dedicated models and other models are based on GSF report. ${ }^{(15)}$ CT Expo and WinDose use Monte Carlo (MC) data sets from GSF report ${ }^{(12,14)}$ while ImPACT Calculator uses the NRPB MC data set ${ }^{(16)}$ but there is good agreement between these MC data sets. ${ }^{(12)}$ All software packages provide effective dose and DLP as final dose reporting. However, CT-Expo and ImPACT calculator provide CTDI $_{\mathrm{w}}$ but WinDose provides CTDI air (also called “air kerma”). A detailed comparison of these three software packages, i.e. CT-Expo 1.5, ImPACT CT patient dosimetry calculator 0.99x and WinDose 2.1a is given in Table 1. ${ }^{(17-19)}$ 
Dose estimations were done for head, chest, abdominal and pelvic examinations based on the parameters recommended by the European guidelines. ${ }^{(15)}$ Estimations were also done separately for software-based adult (170-cm height and $70 \mathrm{~kg}$ weight) male and adult female (160-cm height and $60 \mathrm{~kg}$ weight) phantoms. Tube voltage and pitch for all examinations was set to $120 \mathrm{kVp}$ and 1.0 , respectively. Scan lengths and tube current-time products (mAs) were adjusted to the closest values of $\mathrm{CTDI}_{\mathrm{w}}$ and DLP recommended by the European guidelines. These adjustments were done in order to normalise scanning parameters independent to differences in values of CTDI ${ }_{w}$ and DLP between those software packages. For CT-Expo and ImPACT Calculator software, $\mathrm{CTDI}_{\mathrm{w}}$ and DLP are referred to values displayed on "calculation page" while for WinDose software, $\mathrm{CTDI}_{\mathrm{w}}$ were obtained by dividing DLP with scan length showed on "result page". All values of effective doses from WinDose software were referred to the values estimated using organ-weighting factors of ICRP Report $60 .{ }^{(20)}$

\section{Results}

Details of scan parameters, as well as displayed CTDI and DLP that were used and entered into the software packages to estimate the effective doses for both SSCT and MSCT scanners are listed in Appendix A. Those differences will give approximately the same $\mathrm{CTDI}_{\mathrm{w}}$ (less than $1.8 \%$ deviation) and DLP (less than $4.1 \%$ deviation) values for each examination (see Appendix A).

Table 2 shows the comparison of effective doses in SSCT calculated using these three software packages. For the female phantom, the ImPACT calculator consistently shows the lowest effective doses in all examinations while WinDose gives the highest doses in all examinations except for the chest examination. In the male phantom, there is no consistent trend for the lowest and the highest dose. The head examination in the female phantom shows the highest percentage of coefficient of variance (CV) (23.4\%) while the abdominal 
examination in the male phantom shows the highest percentage of CV (12.5\%). Other examinations for both female and male phantoms show less than $10 \%$ of $\mathrm{CV}$.

Table 3 shows the comparison of effective doses in MSCT calculated using these three software packages. CT Expo consistently shows the highest doses from all examinations for both female and male except for the pelvic examination in male. On the other hand, WinDose shows the lowest doses from all examinations for both female and male except for the pelvic examination in male. The highest percentage of $\mathrm{CV}$ is shown in the pelvic general examination (22.2\%) for female phantom and in the head examination (43.8\%) for male phantom. The lowest percentage of $\mathrm{CV}$ is shown in the abdominal examination (16.4\%) for female phantom and the pelvic examination (10.6\%) for male phantom. All examinations show more than $10 \%$ of CV. On average, results for MSCT examination have higher CV than that for SSCT.

\section{Discussion}

CT dose reporting software packages are used for the convenience of CT users to obtain CT dose descriptors for monitoring the scanning performance and patient dose. Software packages are continuously developed and upgraded to give better dose results in terms of accuracy and user-friendliness. However, this study shows that different software packages output slightly different dose values in the report and the results of calculations are not the same especially for MSCT. Therefore, it is advisable that the researchers must always state the name of the dose reporting software package used and the ICRP report number to estimate effective doses from CT examinations. This is to identify the source of dose estimations so that the results can be used for inter-comparison.

Most current effective dose conversion factors in CT use organ tissue-weightings factors that are derived from the ICRP 60. ${ }^{(20)}$ In 2007, ICRP released the 103rd publication updating the 16-year old ICRP 60 dataset, following the latest available scientific information about the 
biology and physics of radiation exposure. ${ }^{(21)}$ The effective dose in the head and chest CT scans was increased by up to $11 \%$ and $20 \%$, respectively, and decreased by up to $25 \%$ for pelvic scans with use of ICRP 103 weighting factors. ${ }^{(22)}$ Thus, appropriate conversion factors are needed to accurately estimate effective dose.

Both $\mathrm{CTDI}_{\mathrm{vol}}$ and DLP data are available on the picture archiving and communications system as CT scans are being interpreted, thus, it is very convenient for the radiologist or medical imaging technologist to review these data. This allows identification of any unexpected deviation from protocol or technique, equipment malfunction, and unexpectedly high patient doses associated with CT scans. Radiologists can use DLP data from CT scans to estimate patient effective dose, as effective dose puts CT doses into a useful perspective and allows direct comparisons of CT doses with other types of radiological examinations, natural background exposures, and regulatory dose limits. ${ }^{(23)}$

In conclusion, using CT dose reporting packages is an advantage because they are easy to use and produce quick results. However, it must be recognised that there are deviations between the different software packages, and users should understand this and be familiar with different terminologies used in order to provide accurate dose reporting for a consistent comparison. 


\section{References}

1. Brink, J. A., Amis, Jr E. A. Image wisely: a campaign to increase awareness about adult radiation protection. Radiology 257: 601-602 (2010).

2. Sun, Z., Ng, K. H. Multislice CT angiography in cardiac imaging: Part III: radiation risk and dose reduction. Singapore Med. J. 51: 457-463 (2010).

3. Sun, Z., Faridah, Y., Ng, K. H. Coronary CT angiography: how should physicians use it widely and when do physicians request it appropriately? Eur. J. Radiol. (Epub ahead of print) doi:10.1016/j.ejrad.2011.06.040.

4. Goske, M. J., Philips, R. P., Mandel, K., McLinden, D., Racadio, J. M., Hall, S. Image Gently: A web-based practice quality improvement program in CT safety for children. Am. J. Roentgenol. 194: 1177-1182 (2010).

5. Strauss, K. J., Goske, M. J., Frush, D. P., Butler, P. F., Morrison, G. Image Gently vendor summit: working together for better estimates of pediatric radiation dose from CT. Am. J. Roentgenol. 192: 1169-1175 (2009).

6. Sidhu, M., Goske, M. J., Connolly, B., Racadio, J., Yoshiumi, T. T., Strauss, K. J., Coley, B. D., Utley, T. Image Gently, Step lightly: promoting radiation safety in pediatric interventional radiology. Am. J. Roentgenol. 195: W299-301 (2010).

7. Balter, S., Zanzonico, P., Reissm, G. P, Moses J. W. Radiation is not the only risk. Am. J. Roentgenol. 196: $762-767$ (2011).

8. McNitt-Gray, M. F. AAPM/RSNA physics tutorial for residents: topics in CT-radiation dose in CT. RadioGraphics 22: (6): 1541-1553 (2002).

9. U.S. Food and Drug Administration. Provision for alternate measure of the computed tomography dose index (CTDI) to assure compliance with the dose information requirements of the Federal Performance Standard for Computed Tomography. Washington, DC: U.S. Food and Drug Administration, (2006). 
10. ACR CT Accreditation Program. Site scanning instructions for use of the CT phantom for the ACR computed tomography Accreditation program. Available at www.acr.org.

11. Huda, W., Ogden, K. M., Khorasani, M. R. Converting dose-length product to effective dose at CT. Radiology 248: (3) 995 - 1003 (2008).

12. Stamm, G., Nagel, H. D. CT-Expo: A tool for dose evaluation in computed tomography. (Version 1.5). Hannover (2005).

13. ImPACT CT patient dosimetry calculator Excel spreadsheet (version 0.99x, 20 Jan 2006) Available from Imaging Performance and Assessment for Computed Tomography webpage at http://www.impactscan.org/

14. Kalender, W. A., Schmidt, B., Zankl, M., Schmidt, M. A PC program for estimating organ dose and effective dose values in computed tomography. Eur. Radiol. 9: 555562 (1999).

15. European Commission 2000 European guidelines on quality criteria for computed tomography EUR 16262 (Luxembourg: EC).

16. Shrimpton, P. C., Jones, D. G., Hillier, M. C., Wall, B. F., LeHeron, J. C., Faulkner, K. 1991 Survey of CT practice in the UK; Part 2: Dosimetry aspects NRPB-249 (London: HMSO).

17. Zankl, M., Panzer, W., Drexler, G. The calculation of dose from external photon exposures using reference human phantoms and Monte Carlo methods part IV: Organ doses from computed tomographic examinations GSF Report 30/91 (Oberschleißheim: GSF-Forschungszentrum) (1991).

18. Shrimpton, P. C., Jones, D. G., Hillier, M. C., Wall, B. F., LeHeron, J. C., Faulkner, K. Survey of CT practice in the UK; Part 2: Dosimetry aspects NRPB-249 (London: HMSO) (1991). 
19. ICRP 1977 Recommendations of the International Commission on Radiological Protection, ICRP Publication 26 (Oxford: Pergamon).

20. ICRP 1991 Recommendations of the International Commission on Radiological Protection ICRP Publication 60 (Oxford: Pergamon).

21. ICRP 2007 The 2007 Recommendations of the International Commission on Radiological Protection. ICRP publication 103. Ann. ICRP 37 : (2-4): 1-332 (2007).

22. ImPACT . http://www.impactscan.org/ctdosimetry.htm. Published August 28, 2009.

23. Huda, W., Mettler, F. A. Volume CT dose index and dose-length product displayed during CT: what good are they? Radiology 258: 236-242 (2011). 
Appendix A. Comparison of parameters used in the software packages and the values of CTDI and DLP calculated automatically by the software

\begin{tabular}{|c|c|c|c|c|c|c|c|c|c|}
\hline Scanner & Examination & Software package & $\mathrm{kV}_{\mathrm{p}}$ & $\begin{array}{c}\text { Slice thickness / } \\
\text { pitch / scan } \\
\text { mode / beam } \\
\text { collimation }\end{array}$ & mAs & $\begin{array}{l}\text { Scan } \\
\text { length in } \\
\text { cm }\end{array}$ & $\begin{array}{c}\mathrm{CTDI}_{\mathrm{air}} \text { in } \\
\mathrm{mGy} / 100 \mathrm{mAs}\end{array}$ & $\begin{array}{c}\mathrm{CTDI}_{\mathrm{w}} \text { in } \\
\text { mGy/100mAs } \\
\text { (\%deviation) }^{\mathrm{a}}\end{array}$ & $\begin{array}{c}\text { DLP in } \\
\text { mGy.cm } \\
\text { (\%deviation) }^{\mathrm{a}}\end{array}$ \\
\hline \multirow{12}{*}{ SSCT } & \multirow{3}{*}{ Head } & CT Expo & \multirow{24}{*}{120} & \multirow{3}{*}{$\begin{array}{c}5 \mathrm{~mm} / 1.0 \text { / } \\
\text { contiguous / } \\
5 \mathrm{~mm}\end{array}$} & 410 & 17 & - & $59.9(0.2)$ & $1048(0.2)$ \\
\hline & & ImPACT calculator & & & 410 & 17.5 & - & $59.7(0.5)$ & $1045(0.5)$ \\
\hline & & WinDose & & & 485 & 18 & 14.9 & - & $1063.1(1.2)$ \\
\hline & \multirow{3}{*}{ Chest } & CT Expo & & \multirow{9}{*}{$\begin{array}{c}\text { 10mm / } 1.0 \text { / } \\
\text { helical / 10mm }\end{array}$} & 360 & 22 & - & $29.9(0.3)$ & $657(1.1)$ \\
\hline & & ImPACT calculator & & & 380 & 21.5 & - & $30.0(0.0)$ & $646(0.6)$ \\
\hline & & WinDose & & & 400 & 21 & 15.08 & - & $634.8(2.3)$ \\
\hline & \multirow{3}{*}{ Abdomen } & CT Expo & & & 420 & 22 & - & $34.9(0.3)$ & 767 (1.7) \\
\hline & & ImPACT calculator & & & 440 & 22.5 & - & $34.8(0.6)$ & $783(0.4)$ \\
\hline & & WinDose & & & 465 & 22 & 15.08 & - & $770.0(1.3)$ \\
\hline & \multirow{3}{*}{ Pelvis } & CT Expo & & & 420 & 17 & - & $34.9(0.3)$ & $593(4.0)$ \\
\hline & & ImPACT calculator & & & 440 & 16.5 & - & $34.8(0.6)$ & $574(0.7)$ \\
\hline & & WinDose & & & 450 & 16 & 15.08 & - & $558.9(1.9)$ \\
\hline \multirow{12}{*}{ MSCT } & \multirow{3}{*}{ Head } & CT Expo & & \multirow{3}{*}{$\begin{array}{c}\mathrm{mm} / 1.0 / \\
\text { contiguous / } 12 \\
\times 1.5 \mathrm{~mm}\end{array}$} & 320 & 17 & - & $61.1(1.8)$ & $1038(1.1)$ \\
\hline & & ImPACT calculator & & & 320 & 17.5 & - & $60.2(0.3)$ & $1053(0.3)$ \\
\hline & & WinDose & & & 240 & 17 & 15.8 & - & $1029.7(1.9)$ \\
\hline & \multirow{3}{*}{ Chest } & CT Expo & & \multirow{9}{*}{$\begin{array}{c}\text { 10mm / } 1.0 \text { / } \\
\text { helical / } 16 \mathrm{x} \\
1.5 \mathrm{~mm}\end{array}$} & 400 & 22 & - & $30.4(1.3)$ & $664(2.2)$ \\
\hline & & ImPACT calculator & & & 400 & 21.5 & - & $30.4(1.3)$ & $655(0.8)$ \\
\hline & & WinDose & & & 280 & 22 & 15.4 & - & $659.1(1.4)$ \\
\hline & \multirow{3}{*}{ Abdomen } & CT Expo & & & 460 & 22 & - & $35.0(0.0)$ & $769(1.4)$ \\
\hline & & ImPACT calculator & & & 400 & 22.5 & - & $35.0(0.0)$ & $780(0.0)$ \\
\hline & & WinDose & & & 330 & 22 & 15.4 & - & $776.8(0.4)$ \\
\hline & \multirow{3}{*}{ Pelvis } & CT Expo & & & 460 & 16 & - & $35.0(0.0)$ & 559 (1.9) \\
\hline & & ImPACT calculator & & & 400 & 16.5 & - & $35.0(0.0)$ & $578(1.4)$ \\
\hline & & WinDose & & & 310 & 17 & 15.4 & - & $593.6(4.1)$ \\
\hline
\end{tabular}

${ }^{\text {a }}$ percentage of deviation from the values recommended by the European guidelines ${ }^{15}$ 